\title{
Capital social y disponibilidad de mano de obra calificada como impulsores de la competitividad de las empresas que forman parte de clústeres aeroespaciales. El caso de México
}

Ana E. De la Mora ${ }^{(1,2)}$, Gustavo Alarcón(2) y Jesús F. López ${ }^{(2)}$

(1) Escuela de negocios, Universidad de Monterrey, Monterrey México. (e-mail: ana.delamora@udem.edu)

(2) Factultad de Administración y Contabilidad, Universidad Autónoma de Nuevo León, Monterrey - México.

Recibido Mar. 13, 2019; Aceptado May. 6, 2019; Versión final Jul. 23, 2019, Publicado Feb. 2020

\section{Resumen}

El objetivo de la investigación es identificar el impacto del capital social y la disponibilidad de mano de obra calificada en la competitividad de las empresas aeroespaciales que forman parte de clústeres en México. Se aplicó el método de regresión lineal multivariada de mínimos cuadrados ordinarios para el análisis de datos. Los resultados demuestran que el capital social y la disponibilidad de mano de obra calificada son variables que tienen impacto positivo y estadísticamente significativo en la competitividad de las empresas aeroespaciales establecidas en un cluster. Los resultados son relevantes ya que existen pocos estudios empíricos vinculando el captial social con la competitividad de empresas aeroespaciales. Por otro lado, el estudio corrobora la influencia de la disponibilidad de mano de obra calificada en la competitividad de las empresas que forman parte de dicha industria.

Palabras clave: clústeres; industria aeroespacial; capital social; mano de obra calificada; competitividad.

\section{Social capital and qualified labor availability as drivers for competitiveness of enterprises belonging to aerospace clusters. The case of Mexico}

\begin{abstract}
The present study aims to identify the influence of social capital and availability of qualified labor on the competitiveness of aerospace cluster enterprises in Mexico. Data analysis is carried out using multivariate linear regression method of ordinary least squares. The results show that the variables, social capital and the availability of qualified labor, have a positive and statistically significant influence on the competitiveness of aerospace firms established in a cluster. The findings are relevant because there are few empirical studies determining the impact of social capital on the competitiveness of aerospace companies. The study also corroborates the impact of the availability of qualified labor on the competitiveness of the aerospace firms that are part of the clusters.
\end{abstract}

Keywords: clusters; aerospace manufacturing; social capital; qualified labor; competitiveness. 


\section{INTRODUCCIÓN}

La industria aeroespacial genera prosperidad económica en las regiones donde se desarrolla. Esto debido a que promueve empleos altamente calificados y bien remunerados, requiere procesos de manufactura especializados e impulsa la transferencia y creación de conocimiento; por consiguiente tiene impacto positivo en el desarrollo económico. En 2017, la remuneración por persona en la fabricación de equipo aeroespacial fue un $40 \%$ superior a la remuneración de la industria manufacturera general en México (Colección de estudios sectoriales y regionales Conociendo la Industria Aeroespacial, 2018). Así mismo, la industria aeroespacial muestra alto potencial de crecimiento impulsado por el mercado de la aviación comercial y militar, lo cual ha generado mayor demanda de aeronaves para las empresas dedicadas al ensamble, manufactura y producción de componentes. A nivel global la industria aeroespacial ha crecido $3 \%$ a tasa de crecimiento anual compuesto en ingresos del 2010 al 2017 y un 5\% en margen operativo (Deloitte, 2018).

La industria de manufactura aeroespacial se caracteriza por desarrollarse a través de concentraciones o aglomeraciones de empresas dedicadas a este giro e instituciones inter-relacionadas denominadas clústeres. Algunos de los clústeres aeroespaciales en el mundo se han desarrollado de forma natural debido a ciertas condiciones favorables que presenta la región en la que están establecidos, tales como los clústeres europeos de Toulouse Francia o Bavaria en Alemania, los cuales iniciaron de forma natural y han sido fortalecidos por políticas públicas gubernamentales; mientras otros han sido promovidos por los propios gobiernos en búsqueda de consolidar clústeres aeroespaciales que permitan impulsar el desarrollo económico de la región, como es el caso del cluster de Bangalore India o la región Vasca en España.

El concepto de clústeres ha sido ampliamente estudiado desde su introducción por Porter en su teoría sobre la ventaja competitiva de las naciones. Si bien diversos autores han definido este fenómeno, se puede argumentar que coinciden en la definición como un conjunto de empresas y organizaciones interconectadas dentro de un mismo giro económico, que compiten y colaboran, y que son influenciados positivamente por las externalidades de la región donde se generan (Brenner, 2004; Camagni, 2002; Malmberg y Maskell, 1997; Porter, 1990). Uno de los conceptos centrales de la teoría de clústers es la ventaja competitiva o especialización de las regiones. Si los factores de localización geográfica son favorables, se comienza a generar concentraciones de empresas y organizaciones dedicadas a un mismo giro que interactuan entre si, dando dinamismo a la industria. Por lo tanto, las empresas que se establecen dentro de un cluster tendrán una ventaja sobre sus competidores que no se encuentran en clústeres.

Dentro de los factores que se asocian con la formación de clústeres tenemos el clásico modelo dimante de Porter (1998), el cual identifica cinco constructos que influyen en la competitividad de la región: Factores de insumo; instituciones relacionadas y de soporte; condiciones de demanda; contexto para la estrategia de la empresa; así como promoción de inversión y generación de nuevas empresas. La relación de los factores regionales de los clústeres con la competitividad de las empresas, ha sido estudiada en diversas investigaciones y existen modelos teóricos que vincula dichos factores con la competitividad de las empresas; varios de estos modelos tiene su base en la teoría de Porter (Hsu et al., 2013; Porter, 1998). Se observa que los primeros estudios sobre los factores que influyen en la competitividad de las empresas que forman parte de clústeres tienen su enfoque en factores transaccionales o de insumos propios de la región; mientras que estudios recientes dan mayor importancia a factores de colaboración, redes o capital social que se genera entre los participantes del cluster.

\section{Importancia de clústeres aeroespaciales en México}

En México, el sector aeroespacial es considerado uno de los sectores prioritarios dentro de la política pública en materia de desarrollo económico a nivel nacional desde 2010. El producto interno bruto de fabricación de equipo aeroespacial en México tuvo un crecimiento de $151 \%$ del 2010 al 2017 y para este último año tuvo un valor de veintidós mil millones de pesos de acuerdo a cifras del Instituto Nacional de Estadística y Geografía INEGI (2018). En términos de exportaciones, se estima que para el cierre del 2018 sea por más de ocho mil millones de dólares.

Cinco estados de México concentran el $72 \%$ de la producción aeroespacial en el país; en Baja California existen 80 empresas dedicadas al sector, Sonora 51, Chihuahua 36, Querétaro 35 y Nuevo León 33. En lo particular, el Monterrey Aerocluster, objeto del presente análisis, se formaliza como una asociación civil en el 2009 y hasta la fecha sigue operando bajo el esquema colaborativo de triple hélice: iniciativa privada, gobierno y academia (Monterrey Aerocluster 2016). En el 2017, el cluster integró 33 empresas del sector, las cuales generaron más de tres mil empleos directos en el estado y exportaciones de 800 millones de dólares al año, consolidando su posición como centro de manufactura aeroespacial a nivel nacional e internacional.

Si bien se podría pensar que una de las principales ventajas comparativas de México para el desarrollo de clústeres aeroespaciales es el menor costo de la mano de obra en comparación en países desarrollados, en el caso de la manufactura aeroespacial en México ofrece otras ventajas: una de estas ventajas se refiere a la 
disponibilidad de la mano de obra calificada, lo que comprende a jóvenes especializados de nivel técnico y profesional en manufactura avanzada. La mayor parte de la producción de aeronaves y componentes tienen como destino Estados Unidos y Canadá, donde la mano de obra dedicada a la manufactura es cada vez más escasa, esto ocasionado por tres motivos principales: el primero se refiere al crecimiento de la población económicamente activa, la cual se espera tenga un crecimiento del 2015 al 2025 de $23 \%$ en México y para Estados Unidos 4\%. La edad promedio de la fuerza laboral en México es de 35 años promedio mientras que en Estados Unidos es de 47 años; el segundo se refiera a la cantidad de jóvenes egresados de áreas de ingeniería y manufactura sobre todo en la región norte del país, en México cada año se gradúan entre 130 y 150 mil jóvenes de estas áreas de conocimiento, lo cual es una proporción mayor a países como Reino Unido, Italia, España y Brasil por ejemplo. Como tercer factor, cada vez menos jóvenes estadounidenses o canadienses optan por trabajar en un contexto de manufactura. Otra de las ventajas que ofrece México para la industria aeroespacial es la ubicación geográfica, como se mencionó el destino principal de los componentes y aeronaves producidas en México es Estados Unidos y Canadá, dichos componentes son grandes en volumen por lo cual el costo logístico es un factor relevante para la rentabilidad de las empresas aeroespaciales.

\section{Planteamiento del problema}

De acuerdo a la revisión de literatura, se identifican varios factores de impacto en la competitividad de las empresas que forman parte de clústeres en general y en particular de la industria aeroespacial. Entre ellos están: mano de obra calificada, disponibilidad de financiamiento, capital social, infraestructura para la investigación y desarrollo, condiciones para la atracción de inversión extranjera directa, presencia de proveedores especializados y de industrias relacionadas, entre otros. Existen estudios empíricos que vinculan dichos factores con la competitividad de la región o de las empresas, principalmente en regiones tradicionalmente aeroespaciales como son Estados Unidos, Francia, Canadá o Alemania (Brenner y Mühling, 2013; Elola et al., 2012; Lei y Huang, 2014; Steenhuis y Kiefer, 2016). Sin embargo, no existen estudios empíricos que analicen los factores de impacto en la competitividad de empresas que forman parte de clústeres aeroespaciales en México y en Latinoamérica, lo que establece una brecha de conocimiento en regiones emergentes.

Del conjunto de factores regionales con impacto en la competitividad de empresas establecidas en clústeres aeroespaciales que se han identificado, la presente investigación se enfoca en dos de ellos: capital social, ya que se ha definido conceptualmente como un factor de impacto en la competitividad de empresas, sin embargo no existen estudios empíricos que demuestren dicho impacto en empresas de clústeres aeroespaciales. Como segunda variable, la disponibilidad de mano de obra calificada, dada la particular importancia en lo relativo a capital humano en la industria. Por lo tanto, el objetivo de la presente investigación es identificar el impacto que tiene capital social CS y la disponibilidad de mano de obra calificada MOC en la competitividad COM de las empresas del Monterrey Aerocluster, utilizando un método de investigación de tipo cuantitativo.

\section{OTROS ANTECEDENTES}

El concepto de clústeres ha estado presente en la literatura desde los inicios del siglo XX con las aportaciones de autores seminales como Marshall y Webber, quienes identificaron los factores que provocan la aglomeración de empresas de una misma industria y el impacto positivo que tienen en el desempeño de las mismas (Lekachman y Marshall, 1962; Weber, 1929). En la misma línea de pensamiento de dichos autores seminales, Porter (1990) es el primer autor en vincular la competitividad de las naciones con factores propios de la región: los valores, cultura, estructuras económicas, infraestructura, instituciones e historia. Estos factores contribuyen al éxito competitivo de las regiones. En sus primeras aportaciones, Porter (1998) relaciona la competitividad de las empresas con el incremento en la productividad, fortalecimiento de la capacidad de innovación y la generación de nuevas empresas a través del emprendedurismo y la inversión. Más tarde, propone una definición más amplia integrando el valor de los productos y servicios producidos, medidos a través del precio promedio y la eficiencia en la que estos productos se producen (Porter et al., 2009).

Otras definiciones de competitividad de las empresas vinculadas a clústeres incluyen la capacidad de sobresalir en mercados internacionales, medida por la participación de mercado y exportaciones; desempeño operativo y valor económico generado (Chikán, 2008; Feurer y Chaharbaghi, 1994; Hsu et al., 2013; Linde, 2001). Linde (2001) en su meta estudio de 158 clústeres en el mundo define la competitividad de los clústeres participantes como la variable dependiente y la operacionaliza con los ítems: participación de las exportaciones en el mercado global, crecimiento en ventas y habilidad para innovar. Por su parte, Hsu et al. (2013) en su estudio empírico midieron el impacto de los recursos estratégicos de los clústeres en la competitividad de las empresas establecidas en los mismos; para operacionalizar la variable emplearon los 
ítems: incremento en ingresos, utilidad de operación, rentabilidad, competencias tecnológicas, y capacidades para la innovación. La información fue recabada a través de una encuesta que empleaba escala de tipo Likert de 5 puntos. Los ítems considerados para evaluar el desempeño operativo de las empresas fueron de acuerdo a la percepción de los participantes en el estudio. Con base en los estudios mencionados anteriormente, se propone como definición de competitividad de las empresas aeroespaciales establecidas en un cluster como la capacidad para generar valor a través del crecimiento en ventas, exportaciones y margen de operación, así como del incremento de las actividades de innovación y la mejora de la productividad.

Uno de los factores de impacto en la competitividad que se evalúan en la presente investigación es el capital social. La competitividad de las regiones no sólo tiene que ver con la alta concentración de organizaciones de la misma actividad económica, sino con la capacidad para coordinar las relaciones entre los miembros de la región, la cual tiene impacto en la innovación. De acuerdo a Boschma (2004) y Lawson (1999), El capital social se refiere al conjunto de reglas, normas, prácticas, hábitos y leguajes que comparten los miembros del cluster. El capital social se relaciona con la cooperación cívica, la confianza y la infraestructura social. (Boschma, 2004; Lawson, 1999).

Dentro de las teorías principales asociadas al capital social en clústeres se encuentra la de triple hélice identificada por Etzowitz (2003), quien argumenta que la colaboración entre las empresas, la academia y el gobierno es de gran importancia para el fortalecimiento de la competitividad de la región y de las empresas que participan en los clústeres. Estudios posteriores han explicado la relación entre capital social explicado por la colaboración de la triple hélice y la competitividad de clústeres, como el realizado por Mauricio et al. (2017), donde compararon los clústeres vinícolas de Brasil y Chile.

En relación al capital social dentro de los clústeres aeroespaciales, Alfonso-Gil y Vazquez-Barquero (2010) analizan las redes de trabajo en el cluster aeronáutico de Madrid España. Los autores aseveran que al interior de los clústeres aeroespaciales, el capital social tiene un papel fundamental para la transferencia de conocimiento y relaciones comerciales, ya que el acceso a información técnica, aprendizaje interactivo y difusión del conocimiento se facilita cuando existe sólido capital social en el clúster. Sin embargo, no realizan estudio empírico para demostrar la relación entre dichas variables.

Existen pocos estudios empíricos que vinculan el capital social con la competitividad de las empresas. El estudio correlacional propuesto por Cooke et al. (2005) aplicado en empresas pequeñas y medianas, resalta la correlación positiva entre las interacciones entre las personas con la innovación, mejora en la calidad y la participación de mercado. Se observa que la participación en clubs de caridad, sociales y políticos tienen correlación positiva con una mayor participación de mercado y la confianza con las actividades de innovación en las empresa.

Connell y Voola (2013) definen la mercadotecnia relacional como los valores compartidos, empatía, comunicación, unión, reciprocidad y confianza entre los miembros del clúster, una definición muy similar a capital social. El estudio que realizaron se concentró en pequeñas y medianas empresas de manufactura e ingeniería en Australia y se comprobó una correlación positiva entre la mercadotecnia relacional y la ventaja competitiva de las empresas con una $\mathrm{R}^{2}$ de $50 \%$. Para efectos de la presente investigación se define capital social como el conjunto de relaciones de confianza, normas, valores compartidos y actitud de cooperación que se genera entre los individuos, grupos e instituciones, que participan en los clústeres aeroespaciales.

Tomando en consideración la revisión de la literatura relacionada con la variable independiente capital social, se propone como definición conceptual las relaciones de confianza, normas, valores compartidos y las relaciones de cooperación entre empresas, gobierno y academia de acuerdo a la teoría de triple hélice. Los ítems para operacionalizar la variable fueron tomados de estudios empíricos incluidos en la revisión de literatura: existe relación de confianza entre los miembros del cluster, los miembros del cluster comparten normas y principios éticos, existen relaciones de cooperación entre las empresas, existen relaciones de cooperación entre las empresas y la academia, existen relaciones de cooperación entre las empresas y el gobierno, existe cooperación entre los miembros del cluster para realizar proyectos sociales.

En lo que se refiere a los factores regionales que influyen en la competitividad de las empresas que pertenecen a clústeres, la literatura identifica la disponibilidad de mano de obra calificada como uno de ellos. Desde autores seminales como Marshall (1962) y Porter (1998), hasta autores recientes como Elola et al. (2012) y Brenner y Mühling (2013) identifican la disponibilidad de mano de obra calificada como uno de los factores regionales que influyen en la competitividad de las empresas que forman parte de clústeres. Existen estudios empíricos que demuestran la asociación entre la disponibilidad mano de obra calificada o recursos humanos y la competitividad de las empresas que forman parte de clústeres de diversas industrias y regiones. HervásOliver y Albors Garrigos (2007) cuyo estudio tenía el objetivo de identificar el impacto de los recursos y capacidades de los clústeres de azulejos cerámicos de Italia y España en la competitividad de los clústeres, 
midieron el constructo relacionado con recursos humanos con los ítems disponibilidad de trabajadores calificados y el entrenamiento especializado en la región de acuerdo a la percepción de los Directores y Gerentes Generales de las empresas que formaban parte de los clústeres mencionados. El análisis estadístico empleado por los investigadores fue de diferencia de medias, los resultados demuestran que el cluster de Castelón España tiene una media mayor en la competitividad y en entrenamiento especializado que el cluster de Italia teniendo además una diferencia significativa.

Metaxas (2010) en su estudio sobre clústeres del sur de Europa de sectores comerciales, servicios y turismo, identifica las condiciones laborales como una de las variables independientes y la define como: disponibilidad de recursos humanos especializados, buenas relaciones laborales entre los trabajadores y las empresas; así como ética y moral de la fuerza laboral. Un primer análisis fue realizado a través del análisis factorial, donde de un total veintiséis ítems, fueron reducidas a ocho variables. Dentro de estas variables quedó la relacionada con recursos humanos, la cual integró la disponibilidad de recursos humanos especializados, buenas relaciones laborales entre los trabajadores y las empresas; así como ética y moral de la fuerza laboral. A través de un análisis estadístico de tablas de contingencia y Chi Cuadrada, los resultados indican que las condiciones laborales tienen una asociación significativa en la competitividad de las empresas grandes, industriales, especialmente de manufactura.

Por su parte, Hsu et al. (2013) en la región de Taiwán para la industria del software y Zhu y Han (2013) en China para el clúster de aviación; realizaron un estudio explicativo que demuestra la relación causal entre la competitividad y recursos humanos o factores de insumos, donde la mano de obra calificada es una de la variables significativas, encontrando betas de .127 para el ítem de recursos humanos y .914 para el constructo de factores de insumos. En términos de clústeres aeroespaciales en específico, Elola et al. (2013), en su estudio sobre la evolución del cluster aeroespacial Hegan ubicado en la región Vasca de España, identificaron la acumulación de conocimiento y experiencia en el personal de la región en relación al ensamble de motores y materiales compuestos para otras industrias como la automotriz y energía eléctrica, como uno de los factores que influyó en su inicio y desarrollo; sin embargo, no demostraron la relación de causalidad con la competitividad de las empresas aeroespaciales. Adicionalmente, resulta interesante observar como el contar con personal entrenado y calificado impactan en las técnicas de manufactura esbelta y esta a su vez en la eficiencia operativa de las plantas de manufactura, tal como Monge et al. (2013) identificó en su estudio sobre manufactura esbelta en México.

En la presente investigación se identifica como segundo factor de impacto en la competitividad de las empresas, la disponibilidad de mano de obra calificada y se define como la cantidad de mano de obra con habilidades, competencias y conocimientos propios de la industria aeroespacial y relacionadas; suficiencia de egresados de estudios técnicos y profesionales; así como la capacitación especializada disponible.

\section{HIPOTESIS}

De acuerdo con el planteamiento del problema y el marco conceptual, se establece como hipótesis general de la investigación que el capital social CS y la disponibilidad de mano de obra calificada MOC son factores que impactan positivamente en la competitividad COM de las empresas aeroespaciales que forman parte del Monterrey Aerocluster. La hipótesis pretende explicar de forma parcial a través de las dos variables independientes el comportamiento de la variable dependiente ya que existen otros factores que influyen en la misma. Se presenta el modelo esquemático de la hipótesis general:

$$
\mathrm{COM}=b_{0}+b_{1} C S_{+} b_{2} \mathrm{MOC}
$$

Donde la variable dependiente competitividad de las empresas COM, se operacionaliza a través de los ítems: crecimiento en los últimos 3 años en ventas, en margen de operación, en exportaciones, productividad y en actividades de innovación.

La primer variable independiente disponibilidad de mano de obra calificada MOC se operacionaliza a través de los ítems: Suficiencia de egresados de nivel técnico especializado en manufactura aeroespacial, calidad de egresados de nivel técnico especializado en manufactura aeroespacial, suficiencia de egresados de nivel profesional especializado en manufactura aeroespacial, calidad de egresados de nivel profesional especializados en manufactura aeroespacial, calidad de instituciones de educación superior especializadas en áreas de manufactura aeroespacial y calidad de programas de capacitación enfocados a manufactura aeroespacial. La segunda variable independiente capital social CS se operacionaliza a través de los ítems: existen relaciones de confianza entre los miembros del cluster, los miembros del cluster comparten normas y principios éticos, existe relaciones de cooperación entre las personas que forman parte del cluster, existen relaciones de cooperación entre las empresas y el gobierno, existen relaciones de cooperación entre las empresas y la academia y existe cooperación entre los miembros del clúster para realizar proyectos sociales. 


\section{METODOLOGÍA}

Dada la naturaleza del problema de investigación el estudio es de carácter cuantitativo, explicativo, no experimental y transeccional, cuyo principal propósito es conocer el impacto de las variables independientes capital social CS y disponibilidad adecuada de mano de obra calificada MOC y en la dependiente competitividad COM. La población objetivo del estudio son las empresas de manufactura aeroespacial que se encuentran en el Monterrey Aerocluster en México. Los sujetos de estudio son directores y gerentes generales, gerentes de operaciones, calidad, comerciales, recursos humanos y suministros de empresas aeroespaciales. El Monterrey Aerocluster cuenta con 29 empresas dedicada a la manufactura aeroespacial y se obtuvo respuesta de 21 participantes, por lo que la muestra de la investigación incluye 21 encuestas.

La recolección de la información se realizó a través de un cuestionario autoadministrado, el cual se envió por correo electrónico a los miembros del Monterrey Aerocluster. El instrumento fue desarrollado con base en los estudios empíricos que sustentan el marco téorico y validado previamente por expertos de acuerdo la metodología de validez donde se evalua la validez de contenido y de relevancia de los ítems propuestos para operacionalizar las variables. Se evaluó la fiabilidad del cuestionario al aplicar la prueba de Alfa de Cronbach. Los resultados arrojados muestran coeficientes en el rango aceptado, tal como se muestra en la tabla 1.

Tabla 1: Alpha de Cronbach

\begin{tabular}{|l|c|c|}
\hline Variable & Alpha de Cronbach & Número de ítems \\
\hline $\begin{array}{l}\text { COM Competitividad } \\
\text { Variable dependiente }\end{array}$ & .881 & 5 \\
\hline $\begin{array}{l}\text { CS Capital Social } \\
\text { Variable independiente }\end{array}$ & .758 & 7 \\
\hline $\begin{array}{l}\text { MOC Disponibilidad de } \\
\text { Mano de Obra } \\
\text { Variable independiente }\end{array}$ & .865 & 6 \\
\hline
\end{tabular}

En la tabla 2 se listan los ítems considerados en cada una de las variables que conforman el estudio. Para los subsecuentes análisis se mantienen todos los ítems en cada una de las variables. En la misma tabla se muestran los pesos relativos de cada ítem en relación a la variable a la que pertenecen. Sin embargo, para la construcción de la variable se optó por seguir el análisis factorial para eliminar la posible colinealidad.

Tabla 2: Alpha de Cronbach pesos de los ítems y resultado de Alpha si se elimina ítem

\begin{tabular}{|l|c|c|}
\hline & $\begin{array}{c}\text { Correlación corregida de } \\
\text { total de ítems }\end{array}$ & $\begin{array}{c}\text { Alpha de Cronbach si ítem } \\
\text { se elimina }\end{array}$ \\
\hline $\begin{array}{l}\text { Variable dependiente: COM } \\
\text { Crecimiento Ventas CV }\end{array}$ & .758 & .847 \\
\hline Crecimiento margen operación CMO & .908 & .816 \\
\hline Crecimiento exportaciones CEX & .640 & .877 \\
\hline Crecimiento productividad CPRO & .792 & .843 \\
\hline Crecimiento innovación CINN & .558 & .890 \\
\hline Variable independiente: MOC & & .760 \\
\hline Suficiencia egresados técnicos SET & .312 & .718 \\
\hline Calidad egresados técnicos CET & .527 & .760 \\
\hline Suficiencia profesionistas SP & .296 & .705 \\
\hline Calidad profesionistas CP & .620 & .716 \\
\hline Calidad instituciones CI & .547 & .674 \\
\hline Calidad programas capacitación CPP & .698 & .751 \\
\hline Disponibilidad mano de obra calificada DMOC & .381 & \\
\hline Variable independiente: CS & & .826 \\
\hline Relaciones de confianza RC & .747 & .835 \\
\hline Comparten normas, principios éticos CET & .723 & .822 \\
\hline Relaciones de cooperación/ personas RCP & .837 & .882 \\
\hline Cooperación empresas/ gobierno RCG & .476 & .833 \\
\hline Cooperación empresas/ academia RCA & .719 & .857 \\
\hline Cooperación en proyectos sociales CPSO & .590 & \\
\hline
\end{tabular}


Dentro de los métodos de estadística inferencial que se emplearon en la investigación están el análisis factorial de componentes principales, el cuál se aplicó con el propósito de reducir el número de ítems en las variables. El principal método estadístico que se emplea es la regresión lineal multivariada de mínimos cuadrados ordinarios para demostrar la causalidad entre las variables independientes capital social CS y disponibilidad de mano de obra calificada MOC y con la dependiente competitividad COM.

\section{RESULTADOS Y DISCUSIÓN}

Al aplicar el análisis factorial a la variable dependiente competitividad COM, los ítems que conforman la variable: crecimiento en ventas, margen de operación, exportaciones, productividad y actividades de innovación; se agruparon en un solo factor el cual explica el $77 \%$ de la varianza del constructo, tal como se muestra en la tabla 3. Solo se reporta un factor debido a que el Eigenvalue inicial de los factores subsecuentes al primer factor es menor a 1 y el primer factor captura gran parte de la varianza del constructo.

Tabla 3: Resultados análisis factorial variable dependiente COM

\begin{tabular}{|l|r|r|r|r|r|r|}
\hline \multirow{2}{*}{ Componente } & \multicolumn{3}{|c|}{ Eigenvalues iniciales } & \multicolumn{3}{c|}{ Varianza extraída } \\
\cline { 2 - 7 } & \multicolumn{1}{|c|}{ Total } & \% de Varianza & \% Acumulada & Total & \% de Varianza & \% Acumulada \\
\hline 1 & 3.085 & 77.113 & 77.113 & 3.085 & 77.113 & 77.113 \\
2 & .477 & 11.928 & 89.041 & & & \\
3 & .254 & 6.347 & 95.388 & & & \\
4 & .184 & 4.612 & 100.000 & & & \\
\hline
\end{tabular}

Por otro lado, se corrió la prueba de esferidad de Bartlett la cual resulta con una significancia menor a 0.05 por lo tanto las variables no están relacionadas con la población y la prueba Kaisen-Meyer-Olkin que dió un resultado de 0.753 el cual se encuentra entre el rango recomendado de 0.5 y 1 , por lo tanto es adecudado como factor resultantante para la variable dependiente competitividad COM. Para la variable independiente capital social CS, los ítems se agruparon en un solo factor el cual explicó el $63 \%$ de la varianza del constructo, tal como se muestra en la tabla 4. Se considera solo un factor dado que el Eigenvalue inicial de los factores subsecuentes al primero es menor a 1 y el primer factor caputra $63 \%$ de la varianza. Se realizó la prueba de esferidad de Bartlett con un resultado menor a 0.05 y la de $\mathrm{KMO}$ con un resultado de 0.79 por lo que es un factor adecuado.

Tabla 4: Resultados análisis factorial variable independiente CS

\begin{tabular}{|c|c|c|c|c|c|c|}
\hline \multirow{2}{*}{ Componentes } & \multicolumn{3}{|c|}{ Eigenvalues iniciales } & \multicolumn{3}{c|}{ Varianza extraída } \\
\cline { 2 - 7 } & Total & \% de Varianza & \% Acumulada & Total & \% de Varianza & \% Acumulada \\
\hline 1 & 3.784 & 63.060 & 63.060 & 3.784 & 63.060 & 63.060 \\
2 & .872 & 14.541 & 77.601 & & & \\
3 & .512 & 8.527 & 86.128 & & & \\
4 & .455 & 7.576 & 93.704 & & & \\
5 & .220 & 3.671 & 97.375 & & & \\
6 & .157 & 2.625 & 100.000 & & & \\
\hline
\end{tabular}

Así mismo, se aplicó el análisis factorial para la variable independiente disponibilidad de mano de obra calificada MOC, dando como resultado dos constructos que en conjunto explican el $71 \%$ de la varianza como se explica en la tabla 5. Se consideran solo dos factores debido a que el Eigenvalue de los factores subsecuentes es menor a 1 y en conjunto ambos factores explican la mayor parte de la varianza. El primer componente se puede definir como la suficiencia en egresados de nivel técnico, calidad de programas de capacitación y de instituciones educativas, por lo tanto se califica al constructo como capacitación y suficiencia técnicos CAPTEC. El segundo componente se define como la calidad de los egresados de nivel técnico, así como la suficiencia y calidad de los egresados profesionistas, por lo que se identifica como calidad egresados CAL. Se muestran los resultados de las cargas de los ítems en cada uno de los factores mencionados en la tabla 6. De igual forma, se corrió la prueba de esferidad de Bartlett la cual resultó significativa y la de KaisenMeyer-Olkin, la cual arrojó un coeficiente de 0.51 que se encuentra dentro del rango aceptado. 
Tabla 5: Resultados análisis factorial variable independiente MOC

\begin{tabular}{|l|r|r|r|r|r|r|}
\hline \multirow{2}{*}{ Componentes } & \multicolumn{3}{|c|}{ Eigenvalues iniciales } & \multicolumn{3}{c|}{ Varianza extraída } \\
\cline { 2 - 7 } & \multicolumn{1}{|c|}{ Total } & \% de Varianza & \% Acumulada & Total & \% de Varianza & \% Acumulada \\
\hline 1 & 2.759 & 45.986 & 45.986 & 2.759 & 45.986 & 45.986 \\
3 & 1.489 & 24.818 & 70.804 & 1.489 & 24.818 & 70.804 \\
4 & .933 & 15.556 & 86.360 & & & \\
5 & .512 & 8.526 & 94.886 & & & \\
6 & .186 & 3.102 & 97.988 & & & \\
\hline
\end{tabular}

Tabla 6: Matriz de componentes rotados variable independiente MOC

\begin{tabular}{|l|r|r|}
\hline \multirow{2}{*}{} & \multicolumn{2}{|c|}{ Componente } \\
\cline { 2 - 3 } & CAPTEC & \multicolumn{1}{|c|}{ CAL } \\
\hline D1.1SuficienciaTecnicos & .777 & -.005 \\
\hline D1.2.CalidadTecnicos & .303 & .749 \\
\hline D1.3SuficienciaProfesionistas & -.102 & .811 \\
\hline D1.4CalidadProfesinistas & .199 & .899 \\
\hline D1.5CalidadInstituciones & .775 & .107 \\
\hline D1.6CalidadProgramasCapacitacion & .891 & .261 \\
\hline
\end{tabular}

Seguido del análisis factorial, se llevó a cabo el análisis de regresión lineal multivariada con el método enter, incluyendo como variables causales las resultantes del análisis factorial capital social CS, capacitación y suficiencia de técnicos CAPTEC, calidad de egresados CAL y como variable dependiente el factor resultado del análisis factorial competitividad COM. El modelo resultante es un modelo con significancia, sin embargo, como la tabla 7 de coeficientes lo muestra los constructos capacticación y suficiencia de técnicos CAPTEC y calidad de egresados CAL no resultan siginificativos.

Tabla 7: Coeficientes modelo de regresión CAPTEC, CAL y CS variables independientes y COM dependiente

\begin{tabular}{|c|c|c|c|c|c|c|}
\hline \multirow{2}{*}{\multicolumn{2}{|c|}{ Modelo }} & \multicolumn{2}{|c|}{$\begin{array}{l}\text { Coeficientes no } \\
\text { estandarizados }\end{array}$} & \multirow{2}{*}{$\begin{array}{c}\text { Coeficientes } \\
\text { estandarizados } \\
\text { Beta } \\
\end{array}$} & \multirow{2}{*}{$\mathrm{t}$} & \multirow[b]{2}{*}{ Sig. } \\
\hline & & $\mathrm{B}$ & Error estándar & & & \\
\hline \multirow[t]{4}{*}{1} & (Constante) & $1.886 \mathrm{E}-17$ & .192 & & .000 & 1.000 \\
\hline & CAPTEC & -.072 & .231 & -.072 & -.310 & .761 \\
\hline & CAL & -.195 & .218 & -.195 & -.893 & .387 \\
\hline & CS & .757 & .249 & .757 & 3.040 & .009 \\
\hline
\end{tabular}

Posteriormente se corrió la regresión lineal multivariada con el método de pasos sucesivos incluyendo como variable causal el constructo capital social CS y como variable dependiente competitividad COM. EI modelo resultante tiene una $\mathrm{R}^{2}$ del $42 \%$ con grado de significancia estadística, como se muestra en las tablas 8 y 9 . Tomando los resultados de la tabla de coeficientes resultantes del modelo de regresión que se muestran en la tabla 10, se obtiene la ecuación (2):

$\mathrm{COM}=0.650 \mathrm{CS}$

Tabla 8: Resumen modelo de regresión CS y COM

\begin{tabular}{|l|c|r|r|r|}
\hline Modelo & $\mathrm{R}$ & $\begin{array}{c}\mathrm{R} \\
\text { Cuadrada }\end{array}$ & $\begin{array}{c}\text { R Cuadrada } \\
\text { Ajustada }\end{array}$ & $\begin{array}{c}\text { Estimación del } \\
\text { error estándar }\end{array}$ \\
\hline 1 & $.650^{\mathrm{a}}$ & .422 & .386 & .78343842 \\
\hline
\end{tabular}

Tabla 9: ANOVA modelo de regresión CS y COM

\begin{tabular}{|c|c|c|c|c|c|c|}
\hline \multicolumn{2}{|c|}{ Modelo } & $\begin{array}{l}\text { Suma de } \\
\text { cuadrados }\end{array}$ & $\mathrm{gl}$ & Media cuadrada & $\mathrm{F}$ & Sig. \\
\hline \multirow[t]{3}{*}{1} & Regresión & 7.180 & 1 & \multirow{3}{*}{$\begin{array}{r}7.180 \\
.614\end{array}$} & \multirow[t]{3}{*}{11.697} & \multirow[t]{3}{*}{$.004^{b}$} \\
\hline & Residual & 9.820 & 16 & & & \\
\hline & Total & 17.000 & 17 & & & \\
\hline
\end{tabular}


Tabla 10: Coeficientes modelo de regresión CS y COM

\begin{tabular}{|c|c|c|c|c|c|c|}
\hline \multirow{2}{*}{\multicolumn{2}{|c|}{ Modelo }} & \multicolumn{2}{|c|}{ Coeficientes no estandarizados } & \multirow{2}{*}{$\begin{array}{c}\begin{array}{c}\text { Coeficientes } \\
\text { estandarizados }\end{array} \\
\text { Beta }\end{array}$} & \multirow[t]{2}{*}{$\mathrm{t}$} & \multirow[t]{2}{*}{ Sig. } \\
\hline & & $\mathrm{B}$ & Error estándar & & & \\
\hline \multirow{2}{*}{1} & (Constante) & $-4.560 \mathrm{E}-18$ & .185 & & .000 & 1.000 \\
\hline & FacCS & .650 & .190 & .650 & 3.420 & .004 \\
\hline
\end{tabular}

Dado que el modelo resultante obtuvo una $\mathrm{R}^{2}$ moderada, se corrió un segundo modelo de regresión lineal multivariada con el método de pasos sucesivos incluyendo como variables causales los efectos directos de los ítems medibles de los constructos capital social CS y disponibilidad de mano de obra calificada MOC y como variable dependiente el resultado del análisis factorial para competitividad COM. La lista de ítems medibles de cada constructo se presenta en la tabla 11.

Tabla 11: Efectos directos de los ítems que forman parte de variables independientes

\begin{tabular}{|l|l|}
\hline Variable independiente: MOC & Variable independiente: CS \\
\hline Suficiencia egresados técnicos SET & Relaciones de confianza RC \\
\hline Calidad egresados técnicos CET & Comparten normas, principios éticos CET \\
\hline Suficiencia profesionistas SP & Relaciones de cooperación/ personas RCP \\
\hline Calidad profesionistas CP & Cooperación empresas/ gobierno RCG \\
\hline Calidad instituciones Cl & Cooperación empresas/ academia RCA \\
\hline Calidad programas capacitación CPP & Cooperación en proyectos sociales CPSO \\
\hline $\begin{array}{l}\text { Percepción general de disponibilidad mano de obra } \\
\text { calificada DMOC }\end{array}$ & \\
\hline
\end{tabular}

El modelo resultante es un modelo con significancia estadística que incluye los efectos de uno de los ítems considerado en la variable capital social y otro ítem considerado en la variable disponibilidad de mano de obra calificada MOC, con una $\mathrm{R}^{2}$ de $77 \%$ y con significancia estadística, tal como se muestra en las tablas 12 y 13 . Considerando los coeficientes para las variables independientes que se muestran en la tabla 14, se propone la siguiente ecuación que responde a la pregunta de investigación sobre el impacto de las relaciones de cooperación entre empresas y academia RCA, ítem que forma parte de la variable capital social CS y percepción general de disponibilidad de mano de obra calificada DMOC en la competitividad COM de las empresas aeroespaciales. Por útlimo, se realizó la prueba de colinealidad, el modelo no muestra multicolinealidad dado que el coeficiente de condición resultante fue 9, menor al 15 recomendado.

$$
\mathrm{COM}=-3.579+0.488 \mathrm{RCA}+0.318 \mathrm{DMOC}
$$

Donde $\mathrm{RCA}=$ Relaciones de cooperación entre empresas y academia, y DMOC= Percepción general de disponibilidad de mano de obra calificada

Tabla 12: Modelo de regresión ítems de CS y MOC como independientes y COM como dependiente

\begin{tabular}{|l|r|r|r|r|}
\hline Modelo & $\mathrm{R}$ & R Cuadrada & $\begin{array}{c}\text { R Cuadrada } \\
\text { Ajustada }\end{array}$ & $\begin{array}{c}\text { Estimación del } \\
\text { error estándar }\end{array}$ \\
\hline 1 & $.877^{\mathrm{b}}$ & .770 & .739 & .51067228 \\
\hline
\end{tabular}

Tabla 13: ANOVA modelo de regresión ítems de CS y MOC como independientes y COM como dependiente

\begin{tabular}{|c|c|c|c|c|c|c|}
\hline \multicolumn{2}{|c|}{ Modelo } & $\begin{array}{l}\text { Suma de } \\
\text { cuadrados }\end{array}$ & gl & Media cuadrada & $\mathrm{F}$ & Sig. \\
\hline \multirow[t]{3}{*}{1} & Regresión & 13.088 & 2 & 6.544 & 25.094 & $.000^{c}$ \\
\hline & Residual & 3.912 & 15 & .261 & & \\
\hline & Total & 17.000 & 17 & & & \\
\hline
\end{tabular}


Tabla 14: Coeficientes modelo de regresión ítems de CS y MOC como independientes y COM como dependiente

\begin{tabular}{|c|c|c|c|c|c|c|c|c|}
\hline \multirow{2}{*}{\multicolumn{2}{|c|}{ Modelo }} & \multicolumn{2}{|c|}{$\begin{array}{l}\text { Coeficientes no } \\
\text { estandarizados }\end{array}$} & \multirow{2}{*}{$\begin{array}{c}\begin{array}{c}\text { Coeficientes } \\
\text { estandarizados }\end{array} \\
\text { Beta }\end{array}$} & \multirow[t]{2}{*}{$\mathrm{t}$} & \multirow[b]{2}{*}{ Sig. } & \multicolumn{2}{|c|}{$\begin{array}{l}\text { Estadísticos de } \\
\text { colinealidad }\end{array}$} \\
\hline & & $\mathrm{B}$ & Beta & & & & Tolerancia & VIF \\
\hline \multirow[t]{3}{*}{1} & (Constante) & -3.579 & .520 & & -6.881 & .000 & & \\
\hline & $\begin{array}{l}\text { D4.5RelCoopeEmpresaAc } \\
\text { ademia }\end{array}$ & .488 & .096 & .644 & 5.088 & .000 & .956 & 1.046 \\
\hline & D1ManodeObraCalificada & .318 & .085 & .476 & 3.760 & .002 & .956 & 1.046 \\
\hline
\end{tabular}

El principal propósito de la presente investigación ha sido identificar el impacto de los factores regionales capital social CS y disponibilidad de mano de obra calificada MOC en la competitividad COM de las empresas que forman parte del cluster aeroespacial de Monterrey México. Esta problemática surge desde un punto de vista práctico de la necesidad por parte del cluster aeroespacial de profundizar y entender de forma científica el impacto que tienen las actividades que realiza de vinculación y trabajo colaborativo de la triple hélice, así como de los esfuerzos encaminados al desarrollo y atracción de mano de obra calificada en la competitividad de las empresas que forman parte del cluster. Por otro lado, se detecta una brecha de conocimiento al tener pocos estudios empíricos que demuestren causalidad entre las variables independientes capital social CS y disponibilidad de mano de obra calificada con la variable dependiente competitividad. Se aplicó un cuestionario confiable a ejecutivos de nivel directivo y gerencial de empresas de manufactura aeroespacial que forman parte del Monterrey Aerocluster, obteniendo en total 21 encuestas completas de una población total de 29 empresas. Al realizar la prueba estadística de regresión lineal multivariada, los resultados muestran que el constructo formado con análisis factorial de capital social CS tiene un impacto positivo y con significancia estadística en la competitividad COM de las empresas del sector. Este es un hallazgo relevante ya que existen pocos estudios empíricos que han demostrado este impacto entre al capital social CS y la competitividad COM de las empresas que forman parte de un clúster.

Para los clústeres aeroespaciales es de especial relevancia desarrollar relaciones de confianza y cooperación entre las empresas, instituciones gubernamentales y academia, así como compartir normas y principios éticos. Se observa que la transferencia de conocimiento, actividades de innovación y oportunidades comerciales se facilitan con el capital social CS del ecosistema de clústeres, dando como resultado un impacto positivo en al competitividad COM de las empresas, lo cual queda comprobado con los resultados estadísticos del presente estudio. Los resultados del modelo presentado son congruentes y comprueban los estudios teóricos presentados por Alfonso-Gil y Vazquez-Barquero (2010) y Elola et al. (2011) y los estudios empiricos correlacionales de Cooke et al. (2005) y Connell y Voola (2013), los cuales identifican el capital social CS como uno de los factores con correlación en el desarrollo y prosperidad de las empresas establecidas en clústeres. Este resultado tiene un impacto importante para los planes de trabajo y razón de ser del Monterrey Aerocluster dado que la mayoría de las actividades que realizan como parte de sus comités de trabajo van encaminadas a propiciar relaciones de colaboración entre la triple hélice, con estos resultados se cuenta con el fundamento científico que dichas actividades tienen un impacto positivo y estadísticamente significativo en la competitividad COM de las empresas que foman parte del cluster.

En un segundo modelo de regresión lineal multivariada, donde se consideran como variables causales los ítems que forma parte de las variables capital social CS y disponibilidad de mano de obra calificada MOC, se demuestra que las relaciones de cooperación entre las empresas RCA y la academia, el cual es uno de los ítems que conforman la variable capital social CS, así como la percepción general de disponibilidad de mano de obra calificada DMOC tienen un impacto positivo y con significancia estadística en la competitividad COM de las empresas de manufactura aeroespacial.

La relación de cooperación entre las empresas y la academia RCA como variable causal de la competitividad COM de las empresas aeroespaciales, resulta un hallazgo relevante para la teoría de clústeres porque no se tiene registro de estudios empíricos relacionando esta variable con la competitividad COM de las empresas. En la práctica, dentro del Monterrey Aerocluster existe una fuerte sinergia entre el sector privado y la academia a través de actividades y proyectos de colaboración, lo cual se demuestra científicamente mediante el modelo obtenido.

Por otro lado, se reiteran los hallazgos de Linde (2003), Hervás-Oliver y Albors Garrigos (2007) y Metaxas (2010) donde vinculan como variable causal percepción general de disponibilidad de mano de obra calificada DMOC en la competitividad COM de las empresas establecidas en clústeres en general. De igual forma, el modelo es congruente con los hallazgos de Zhu y Han (2013) en relación al impacto positivo y significativo del factor de insumos de producción, donde está incluido el ítem de mano de obra calificada, en la competitividad de clústeres aeroespaciales. Para los miembros del Monterrey Aerocluster resulta relevante 
comprobar que contar con suficientes profesionistas y técnicos especializados y con las competencias necesarias en la industria aeroespacial, así como capacitación de calidad tiene un impacto positivo y estadísticamente significativo en la competitividad COM de las empresas. Gran parte de los proyectos desarrollados dentro del cluster en conjunto con la academia tienen que ver con la capacitación y desarrollo del personal para laborar en la industria aeroespacial. En este rubro falta mucho por hacer dado que la transformación de manufactura hacia la manufactura 4.0 implicará la necesidad de contar con mano de obra mucho más orientada a diseño, investigación y desarrollo y automatización de procesos a través del uso de tecnología.

\section{CONCLUSIONES}

A partir de los resultados obtenidos se pueden puntualizar las siguientes conclusiones: 1) El estudio demuestra el impacto positivo y estadísticamente significativo entre el capital social CS y la competitividad COM de las empresas que forman parte de clústeres aeroespaciales; 2) La relación de cooperación entre empresa y academia RCA que se desarrollan dentro del cluster, así como la percepción general de disponibilidad de mano de obra calificada DMOC son variables con impacto positivo y estadísticamente significativo en la competitividad COM de las empresas que forman parte de clústeres aeroespaciales; 3) La investigación aporta resultados de un estudio empírico realizado en un país emergente latinoamericano a la literatura clústeres aeroespaciales y es congruente con otros estudios teóricos y empíricos realizados en materia de clústeres; 4) Los hallazgos son útiles para el diseño de programas y proyectos que se realicen en los clústeres aeroespaciales, encaminados al desarrollo de las capacidades, competencias y conocimiento de los profesionistas y técnicos de la industria, así como de las redes de colaboración entre las empresas, academia e instituciones gubernamentales, con el objetivo de impulsar la competitividad de las empresas aeroespaciales.

\section{REFERENCIAS}

Alfonso-Gil, J. y A. Vazquez-Barquero, Networking and Innovation: Lessons from the aeronautical clusters of Madrid, International Journal of Technology Management, ISSN 0267-5730, 50, 337-355 (2010)

Boschma, R., Competitiveness of Regions from an Evolutionary Perspective, doi: 0.1080/0034340042000292601, Regional Studies, 38(9), 1001-1014 (2004)

Brenner, T. y A. Mühling, Factors and Mechanisms Causing the Emergence of Local Industrial Clusters : A Summary of 159 Cases, doi: 10.1080/00343404.2012.701730, Regional Studies, 47(4), 480-507 (2013)

Brenner, T., Local Industrial Clusters: Existance, emerge and evolution, $1^{\text {a }}$ Ed, pp. 8-67, Routledge, New York, USA (2004)

Camagni, R., On the Concept of Territorial Competitiveness: Sound or Misleading?, doi: 10.1080/0042098022000027022 Urban Studies, 39(13), 2395-2411 (2002)

Chikán, A., National and firm competitiveness: a general research model, doi: 10.1108/10595420810874583, Competitiveness Review, 18(1/2), 20-28 (2008)

Colección de estudios sectoriales y regionales Conociendo la Industria aeroespacial, recuperado a partir de www.inegi.org.mx, (2018)

Connell, J. y R. Voola, Knowledge Integration and Competitiveness: a Longitudinal Study of an Industry Cluster, doi: 10.1108/13673271311315178, Journal of Knowledge Management, 17(1), 208-225 (2013)

Cooke, P., N. Clifton, y M. Oleaga, Social Capital, firm embeddedness and regional development, doi: 10.1080/00343400500328065, Regional Studies, 39(8), 1065-1077 (2005)

Deloitte. 2018 Global aerospace and defense industry financial performance study. Recuperado a partir de https://www2.deloitte.com/global/en/pages/manufacturing/articles/gx-mnfg-aerospace-and-defense-finanperformance.html (2018)

Elola, A., J. M. Valdaliso y S. López, The Competitive Position of the Basque Aeroespatial Cluster in Global Value Chains: A Historical Analysis, doi: 10.1080/09654313.2013.733851, European Planning Studies, 21(7), 1029-1045 (2013)

Elola, A., J.M. Valdaliso y otros dos autores, Cluster Life Cycles, Path Dependency and Regional Economic Development: Insights from a Meta-Study on Basque Clusters, doi: 10.1080/09654313.2012.650902 European Planning Studies, 20(2), 257-279 (2012)

Etzkowitz, H., University-Industry-Government Relations Innovation in Innovation: The Triple Helix, doi: 10.1177/05390184030423002, Social Science Information, 42, 293-337 (2003)

Feurer, R. y K. Chaharbaghi, Management Decision Defining Competitiveness: A Holistic Approach, doi: 10.1108/00251749410054819, Management Decision International Journal of Operations, 32(6), 49-58 (1994) 
Han, Q. y Y. Zhu, Research on Grey Relational Evaluation of the Competitiveness of Aviation Industrial Cluster: By taking Xi'an Yanliang Aviation Park as an example, doi: 10.1109/GSIS.2013.6714781, Proceedings of IEEE International Conference on Grey Systems and Intelligent Services, GSIS, 25(4), 225-229 (2013)

Hsu, M.-S., Y. Lai y F. Lin, Effects of Industry Clusters on Company Competitiveness: Special Economic Zones in Taiwan, doi: 10.1142/S0219091513500173, Review of Pacific Basin Financial Markets and Policies, 16(03), 1350017 (2013)

Lawson, C., Towards a Competence Theory of the Region, doi: 10.1093/cje/23.2.151, Cambridge Journal of Economics, 23(2), 151-166 (1999)

Lei, H. y C.H, Huang, Geographic clustering, network relationships and competitive advantage Two industrial clusters in Taiwan, doi: 10.1108/MD-08-2013-0426. Management Decision Journal of Business, 52(1), 852-871 (2014)

Lekachman, R., y A. Marshall, Principles of Economics, doi: 10.2307/2145893, Political Science Quarterly (Vol. 77), (1962)

Linde, C. Van Der, The Demography of Clusters — Findings from the Cluster Meta-Study. Harvard Business Review, 130$149(2001)$

Malmberg, A. y P. Maskell, Towards an explanation of regional specialization and industry agglomeration, doi: 10.1080/09654319708720382, European Planning Studies, 5(1), 25-41, (1997).

Maurício, J., B.G. Boaventura y otros tres autores. Triple Helix influence on competitiveness factors: comparison between wine clusters in Brazil and Chile. doi: 10.18568/1980-4865.12343-60, Review of International Business, 12(3), 43-60 (2017)

Metaxas, T., Local Characteristics and Firm Competitiveness in Southeastern Europe: A Cluster Analysis, Journal of Economic and Social Reserach, ISSN 1302-1060, 12(2), 1-39, (2010)

Monge, C., J. Cruz y F. López, Impacto de la Manufactura Esbelta, Manufactura Sustentable y Mejora Continua en la Eficiencia Operacional y Responsabilidad Ambiental en México. doi: 10.4067/S0718. Información tecnológica, 24(4), 5-6 (2013)

Monterrey Aerocluster, Plan estratégico Monterrey Aerocluster, (2016)

Porter, M. E, The Competitive Advantage of Nations, Marzo-Abril, Harvard Business Review, (1990)

Porter, M. E, Cluster and the New Economics of Competition, doi: 10.1042/BJ20111451, Harvard Business Review, November-December, 77-90 (1998)

Porter, M. E., M. Delgado, y otros dos autores, Moving to a New Global Competitiveness Index, Gobal Competitiveness Report 2008/2009, Foro Económico Mundial, pp 43-63, Ginbebra Suiza, (2009)

Steenhuis, H.J. y D, Kiefer, Early Stage Cluster Development: a Manufacturers-led approach in the Aircraft Industry, doi: 10.1108/CR-10-2014-0035, Competitiveness Review, 26(1), 41-65 (2016)

Weber, A., Afred Weber'S Theory of the Location of Industries, pp. 17-34, University of Chicago, Chicago, USA (1929). 